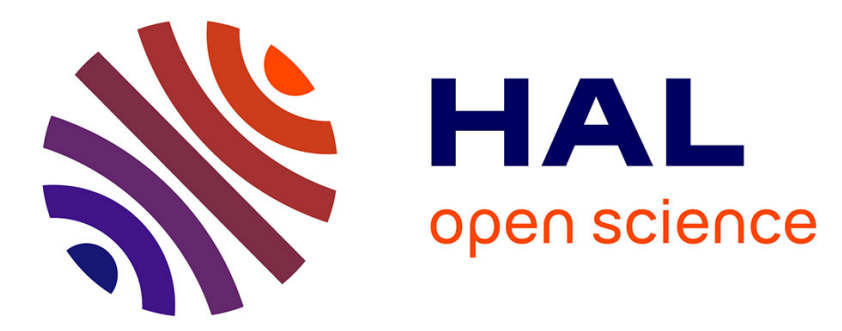

\title{
Scattering effects induced by imperfections on an acoustic black hole placed at a structural waveguide termination
}

Vivien Denis, Adrien Pelat, François Gautier

\section{- To cite this version:}

Vivien Denis, Adrien Pelat, François Gautier. Scattering effects induced by imperfections on an acoustic black hole placed at a structural waveguide termination. Journal of Sound and Vibration, 2016, 362, pp.56-71. 10.1016/j.jsv.2015.10.016 . hal-01288280

\author{
HAL Id: hal-01288280 \\ https://hal.science/hal-01288280
}

Submitted on 14 Mar 2016

HAL is a multi-disciplinary open access archive for the deposit and dissemination of scientific research documents, whether they are published or not. The documents may come from teaching and research institutions in France or abroad, or from public or private research centers.
L'archive ouverte pluridisciplinaire HAL, est destinée au dépôt et à la diffusion de documents scientifiques de niveau recherche, publiés ou non, émanant des établissements d'enseignement et de recherche français ou étrangers, des laboratoires publics ou privés. 


\title{
Scattering effects induced by imperfections on an Acoustic Black Hole placed at a structural waveguide termination
}

\author{
V. Denis ${ }^{1}$, A. Pelat ${ }^{1}$, F. Gautier ${ }^{1}$ \\ Laboratoire d'Acoustique de l'Université du Maine, UMR CNRS 6613, Avenue Olivier \\ Messian, 72085 Le Mans, Cedex 09
}

\begin{abstract}
The so-called "acoustic black hole" effect $(\mathrm{ABH})$ is a passive vibration control technique based on the flexural waves properties in thin structure of varying thickness. A usual implementation consists in using a plate with tapered extremity with a power-law profile, covered with a thin damping layer. The inhomogeneity of the structure leads to a decrease of flexural wave speed and an increase of their amplitude, therefore resulting in an efficient energy dissipation if damping layer is placed where the thickness is minimal. The manufacture of an efficient extremity is difficult because of the small thickness, and often generates imperfections and tearing. Moreover, previous works suggest multiple that flexural modes are propagating across the width of the ABH tip. A model of an ABH multimodal waveguide taking into account an imperfect termination is developed. It shows that an elementary imperfection can affect the reflection coefficient of the extremity and reduce it. Scattering and propagation properties of the extremity
\end{abstract}

Email address: vivien.denis@univ-lemans.fr (V. Denis) 
are also studied. An incident mode excites several modes that are localised in the tapered region and local resonances explain the drops in the reflection coefficient. Experimental evidence of the influence of the imperfection on the reflection coefficient is provided. A key result of the paper is that manufacturing imperfections are not detrimental to the $\mathrm{ABH}$ effect.

Keywords:

reflection coefficient, wave method, flexural vibration, damping, acoustic black hole, inhomogeneous structural waveguide, imperfections, scattering

\section{Introduction}

The control of unwanted vibrations is important for reliability, stability and comfort in many industrial applications [1]. Indeed, vibrations can generate radiated noise [2] but are also a source of structural damage [3]. Most 5 of the classical vibration control methods involve surface damping treatment. The efficiency of these methods has been widely proven, but a major drawback is that they involve an increased mass of the treated structure. This is a concern in many engineering domains for economical or ecological cost. The development of passive vibration control techniques without added mass 10 is interesting in this matter.

The Acoustic Black Hole (ABH) effect $[4,5,6]$ is a passive vibration control technique taking advantage of bending wave properties in structures of decreasing thickness in order to attenuate the reflections at the edge, and consequently decreasing the resonant behaviour. An example of such bound15 ary damping is given in the pioneer work of Vemula et al. [7] which proposes 
to use a graded impedance interface at the edges of a beam, consisting in the association of several pieces of beams made with different materials. The results show that lower reflectivity is caused by energy dissipation within the composite material at the free end coupled with relatively large amplitude vibrations caused by the impedance gradation. In the ABH effect, as first described by Mironov [8], the thickness of a beam edge decreasing smoothly to zero cause flexural waves to slow down and stop without being reflected. The condition of sufficient smoothness can be fulfilled by a power-law thickness profile $h(x)$ in the form:

$$
h(x)=\epsilon x^{m},
$$

where $x$ is the spatial coordinate and $m \geq 2$ (see Fig. 1(a)). If the thickness is strictly zero at the edge [8], it can be shown that the time taken by a wave to reach the edge becomes infinite. For a practical structure with a finite thickness, the reflection coefficient tends to zero with the decrease of the residual thickness at the tip of the tapered profile. It is however shown that manufacturing processes are such that this residual thickness can never be small enough for the effect to be attractive.

As it is shown by Krylov et al. [4, 5] in the framework of geometrical acoustics, the negative effect of the finite thickness at the edge can be compensated by covering the profile with a thin damping layer. A low reflection coefficient can be obtained for the ABH termination. This model has been refined by Georgiev et al. [6] by using a Euler-Bernoulli beam modelling. In this work, reflection and impedance matrices along the tapered beam are computed solving a Riccati equation. Practical rules for determining the op- 
timal geometrical and material properties of the damping layer [9] are found using this model. Further investigations from Denis et al. [10] show that the small thickness of the extremity induces a local plate behaviour in a beam with an $\mathrm{ABH}$ extremity, and that a two-dimensional behaviour has to be taken into account to model the tapered zone; local transverse eigenmodes can be found in the beam structure. Note that two-dimensional ABH have also been described in the literature: circular ABH used as plate vibration damper has been firstly proposed by Gautier et al. [11] and studied both experimentally $[11,12,13]$ and theoretically $[14,15,16]$.

Most of the literature $[4,6,10]$ considers a perfect tapered extremity: the free edge is considered straight and normal in the direction of propagation $x$. The consequence is that an incident plane wave propagating along the $x$-axis remains plane and that the reflected wave is also stricly plane. Thus there should be no excitation of the trapped eigenmodes mentioned in [10]. However the practical realisation fails the assumption of perfect edge because the manufacture of such small thicknesses is difficult and leads to irregular and teared extremities (see Figs. 1(b) and (c)). Moreover, it is observed that the wave field is not unidimensional for some frequencies $[17,18]$. Bowyer et al. [12] study experimentally imperfections of $\mathrm{ABH}$ by comparing an imperfect termination and a shorter thus thicker perfect termination; From this experimental comparison, they conclude that even imperfect, a thinner extremity has a better damping performance. It is proposed in this paper to observe what effects are induced by imperfections for a given thickness at the end to gain design insight needed for enhancing and optimizing $\mathrm{ABH}$ performance. 
A model of the inhomogeneous plate seen as a multimodal waveguide is developed in Sec. 2. It is numerically solved in order to compute the couplings in the waveguide. Sec. 3 presents typical results from the model and proposes an explanation for the phenomena that are observed. Sec. 4 presents an experimental observation of the effect of imperfections. Some conclusions are given in Sec. 5.

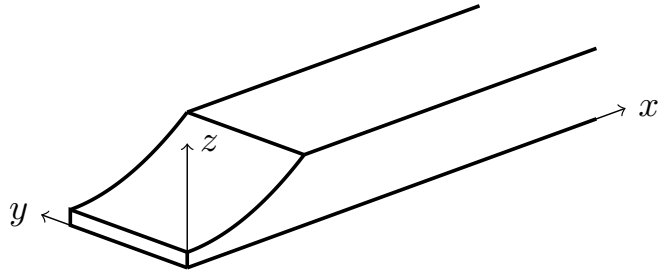

(a)

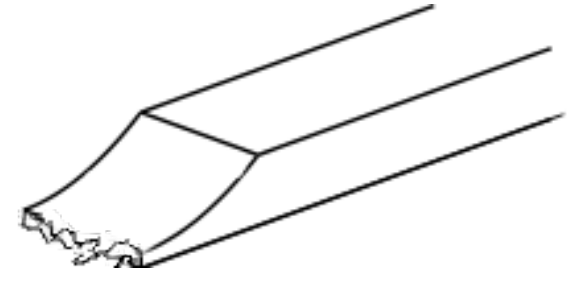

(b)

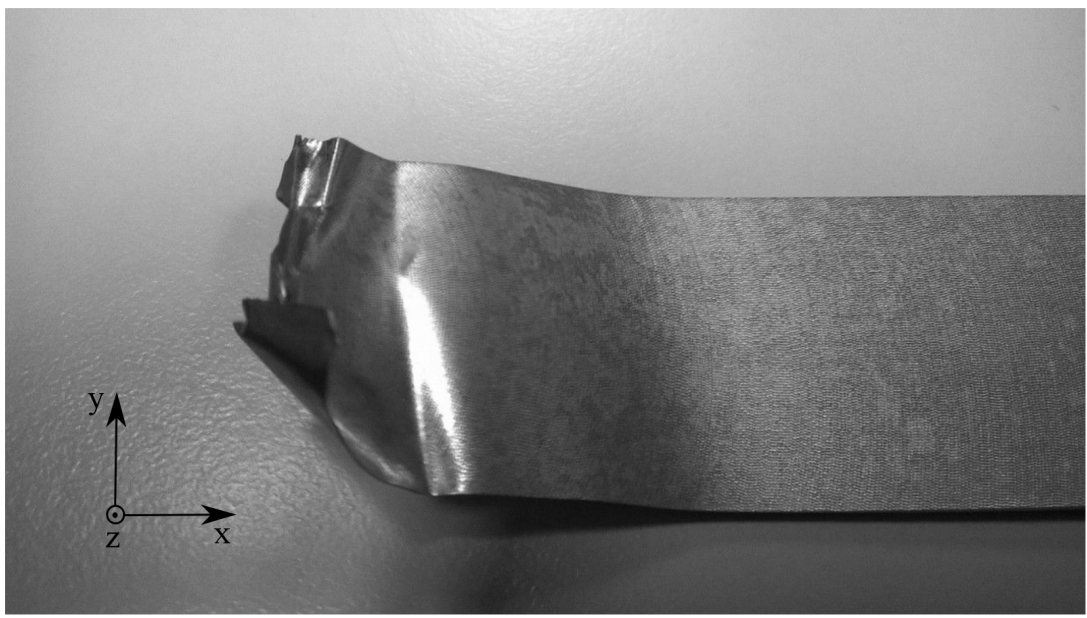

(c)

Figure 1: (a) Nominal shape of the ABH termination (perspective view), (b) scheme of imperfect $\mathrm{ABH}$ termination and (c) picture of imperfections taking place at the tip of an $\mathrm{ABH}$ extremity (top view). Deformations come from stress relaxation during machining. 


\section{Model of an imperfect $\mathrm{ABH}$ termination}

\subsection{Problem statement}

The ABH extremity (see Fig. 2) is considered as an inhomogeneous structural waveguide. In order to describe coupling phenomena between guided modes at the extremity from imperfect edge conditions and to extend the study of the reflection coefficient made in [19], the aim is to obtain the reflection matrix of the extremity or of a region of the waveguide. This reflection matrix can be obtained by either the knowledge of the impedance matrix of the waveguide when it is ended with a free boundary condition, or as part of the scattering matrix of a region of the waveguide. We propose in this section to compute numerically the scattering of a waveguide region.

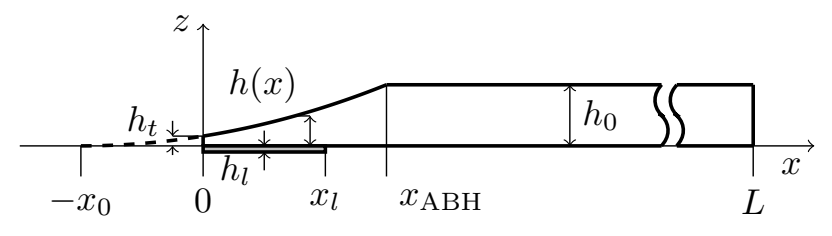

(a)

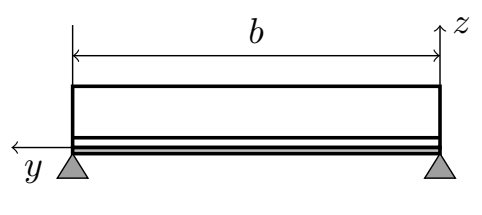

(b)

Figure 2: Model of plate with ABH extremity: edges along $x$ are simply supported, edges along $y$ are free. (a) Side view and (b) front view.

Bending stiffness $D(x)$ and thickness $h(x)$ describe the waveguide and their expressions are given by (see Fig. 2)

$$
D(x)=\frac{E h(x)^{3}}{12 \rho\left(1-\nu^{2}\right)}(1+\mathrm{j} \eta(x)),
$$

where $E, \rho$ and $\nu$ are the Young's modulus, mass density and Poisson ratio of the plate material, respectively, and $\eta(x)$ is the loss factor, and the thickness 
is

$$
h(x)=\left\{\begin{array}{l}
h_{0} \frac{\left(x+x_{0}\right)^{m}}{\left(x_{0}+x_{\mathrm{ABH}}\right)^{m}} \quad \text { if } \quad x \leq x_{\mathrm{ABH}} \\
h_{0} \text { if } \quad x>x_{\mathrm{ABH}}
\end{array}\right.
$$

The effect of the viscoelastic layer can be modelled by an equivalent loss factor [20] which depends on $x$ via [4]

$$
\eta(x)=\left\{\begin{array}{l}
\eta_{p}, \quad \text { if } \quad x \leq x_{l}, \\
\eta_{p}+\eta_{l} \frac{3 E_{l} h_{l}}{E h(x)}, \quad \text { if } \quad x>x_{l},
\end{array}\right.
$$

where $\eta_{p}$ is the loss factor of the plate, and $E_{l}, \eta_{l}, h_{l}$ the Young's modulus,

the loss factor and the thickness of the viscoelastic layer, respectively.

\subsection{Governing equations}

The governing equations for the flexural motion of plate with inhomogeneities along the longitudinal $x$-direction (plate parameters remains invariant along transverse $y$-direction), and in harmonic regime at pulsation

${ }_{95} \omega$ (with the $e^{\mathrm{j} \omega t}$ time convention) give the relations between variables of displacement $w$, slope $\theta_{x}$, bending moment $M_{x}$ and effort $V_{x}$ and their firstorder partial derivatives with respect to $x$ [21]. After several derivations, the following relations are obtained:

$$
\begin{gathered}
\frac{\partial w}{\partial x}=\theta_{x}, \\
\frac{\partial \theta_{x}}{\partial x}=-\frac{1}{D} M_{x}-\nu \frac{\partial^{2} w}{\partial y^{2}}, \\
\frac{\partial M_{x}}{\partial x}=V_{x}-2(1-\nu) D \frac{\partial^{2} \theta_{x}}{\partial y^{2}},
\end{gathered}
$$




$$
\frac{\partial V_{x}}{\partial x}=-\rho h \omega^{2} w+D\left(1-\nu^{2}\right) \frac{\partial^{4} w}{\partial y^{4}}-\nu \frac{\partial^{2} w}{\partial y^{2}}
$$

For simplicity, the two edges along $x(y=0$ and $y=b)$ are simply supported, allowing to write a Levy-type analytical solution [22, 23, 24] for Eqs. (5)-(8) as a product of beam functions; this cannot properly be done assuming free 105 conditions on the four edges [21]. Such lateral boundary conditions define the waveguide which will be studied. This waveguide is represented on Fig. 2(c) and is called a Levy-waveguide. The boundary condition is not realistic for general insight on beam $\mathrm{ABH}$ applications at low frequencies, but is a reasonable condition for high frequencies. Therefore, this choice rules out a direct comparison with the experiment made with beams but allows a multimodal analysis of the effects of the imperfection. The two edges along $y(x=0$ and $x=L)$ are free. The boundary condition of the edge $x=0$ is modified in Sec. 2.5 for taking into account the imperfect extremity.

Each variable $g(x, y)$ (standing for $w, \theta_{x}, M_{x}$ and $V_{x}$ ) is then written as the multimodal expansion

$$
g(x, y)=\sum_{q=1}^{\infty} g_{q}(x) \Psi_{q}(y)
$$

where

$$
\Psi_{q}(y)=\sqrt{2} \sin \left(\frac{q \pi}{b} y\right)
$$

are the orthogonal modes of a simply supported beam [25] also called the transverse modes of the waveguide.

The projection of Eqs. (5)-(8) on the transverse modes yields a set of 
120

projected equations that can be rewritten as the following state equation

$$
\frac{\mathrm{d}}{\mathrm{d} x} \mathbf{W}(x)=\mathbf{H}(x) \mathbf{W}(x)
$$

where the state vector $\mathbf{W}(x)$

$$
\mathbf{W}(x)=\left[\begin{array}{c}
\mathbf{w}(x) \\
\theta_{\mathbf{x}}(x) \\
\mathbf{M}_{\mathbf{x}}(x) \\
\mathbf{V}_{\mathbf{x}}(x)
\end{array}\right],
$$

is the concatenation of displacement, slope, bending moment and force modal vectors. For example, if we consider that the series expansion in Eq. (9) is truncated with $K$ terms, we have:

$$
\mathbf{w}(x)={ }^{\mathrm{T}}\left[w_{1}(x), w_{2}(x), \ldots, w_{K}(x)\right]
$$

125 Matrix $\mathbf{H}$ embeds the plate equations and is expressed as

$$
\mathbf{H}(x)=\left[\begin{array}{ll}
\mathbf{H}_{1}(x) & \mathbf{H}_{2}(x) \\
\mathbf{H}_{3}(x) & \mathbf{H}_{4}(x)
\end{array}\right],
$$

with

$$
\begin{gathered}
\mathbf{H}_{1}(x)=\left[\begin{array}{cc}
\mathbb{O} & \mathbb{I} \\
-\nu \mathbf{I}_{2} & \mathbb{O}
\end{array}\right], \quad \mathbf{H}_{2}(x)=\left[\begin{array}{cc}
\mathbb{O} & \mathbb{O} \\
-\mathbb{I} / D & \mathbb{O}
\end{array}\right], \\
\mathbf{H}_{3}(x)=\left[\begin{array}{cc}
\mathbb{O} & -2(\nu-1) D \mathbf{I}_{\mathbf{2}} \\
-\rho h \omega^{2} \mathbb{I}+\left(1-\nu^{2}\right) D \mathbf{I}_{\mathbf{4}} & \mathbb{O}
\end{array}\right], \quad \mathbf{H}_{4}(x)=\left[\begin{array}{cc}
\mathbb{O} & \mathbb{I} \\
-\nu \mathbf{I}_{2} & \mathbb{O}
\end{array}\right],
\end{gathered}
$$


where $\mathbb{I}$ is the $K \times K$ identity matrix, $\mathbb{O}$ is the $K \times K$ zero matrix and $I_{2 q j}$ and $I_{4 q j}$ are defined as

$$
\begin{aligned}
& I_{2 q j}=-\left(\frac{q \pi}{b}\right)^{2} \delta_{q j}, \\
& I_{4 q j}=\left(\frac{q \pi}{b}\right)^{4} \delta_{q j} .
\end{aligned}
$$

Moreover, the local impedance matrix $\mathbf{Z}(x)$ can be defined as

$$
\left[\begin{array}{c}
\mathbf{M}_{\mathbf{x}}(x) \\
\mathbf{V}_{\mathbf{x}}(x)
\end{array}\right]=\mathrm{j} \omega \mathbf{Z}(x)\left[\begin{array}{c}
\mathbf{w}(x) \\
\theta_{\mathbf{x}}(x)
\end{array}\right] .
$$

Substituting Eq. (17) in Eq. (11), a Riccati non-linear equation is obtained

for the impedance matrix $[26,6]$ :

$$
\frac{\partial \mathbf{Z}(x)}{\partial x}=-\mathbf{Z}(x) \mathbf{H}_{\mathbf{1}}(x)-\mathrm{j} \omega \mathbf{Z}(x) \mathbf{H}_{\mathbf{2}}(x) \mathbf{Z}(x)+\frac{\mathbf{H}_{\mathbf{3}}(x)}{\mathrm{j} \omega}+\mathbf{H}_{\mathbf{4}}(x) \mathbf{Z}(x) .
$$

The boundary value problem is transformed into an initial value problem. The free boundary condition translates into the initial condition $\mathbf{Z}(x=0)=$ (1).

\subsection{Wave expansion of the state vector}

Eigenspace of matrix $\mathbf{H}(x)$ is described by $4 K$ eigenvalues and their associated eigenvectors. The relation between them is

$$
\mathbf{E}(x) \mathbf{H}(x)=\mathbf{\Lambda}(x) \mathbf{E}(x),
$$

where $\boldsymbol{\Lambda}(x)$ is the diagonal matrix containing the eigenvalues of $\mathbf{H}(x)$ on its diagonal and

$$
\mathbf{E}(x)=\left[\begin{array}{ll}
\mathbf{E}_{1} & \mathbf{E}_{2} \\
\mathbf{E}_{3} & \mathbf{E}_{4}
\end{array}\right] \text {, }
$$


with $\mathbf{E}_{\mathbf{1}}$ to $\mathbf{E}_{\mathbf{4}}$ being $2 K \times 2 K$ matrices, is a matrix whose columns are the associated eigenvectors; $\mathbf{E}$ is also the transition matrix between eigenspace and state space. The change of basis is applied using the relation

$$
\mathbf{W}(x)=\mathbf{E}(x) \mathbf{V}(x),
$$

where $\mathbf{V}(x)$ is the wave vector, describing for each mode $q$ the propagating and attenuating waves travelling towards $x>0$ and $x<0$.

For the sake of simplicity, let us first assume a single transverse mode $q$

145 verifying the dispersion equation

$$
k_{q}^{4}(x)=-k_{f}^{4}(x)+\left(\frac{q \pi}{b}\right)^{4}
$$

where $k_{f}=\sqrt[4]{\omega^{2} \rho h / D}$ is the flexural wave number and $k_{q}$ is the guide wave number. Then the eigenvalue matrix $\boldsymbol{\Lambda}$ of $\mathbf{H}$ writes:

$$
\boldsymbol{\Lambda}=\operatorname{diag}\left(j k_{1 q}, k_{2 q},-j k_{1 q},-k_{2 q}\right),
$$

where

$$
\begin{aligned}
& k_{1 q}=\sqrt{k_{f}^{2}-(q \pi / b)^{2}}, \\
& k_{2 q}=\sqrt{k_{f}^{2}+(q \pi / b)^{2}} .
\end{aligned}
$$

It can easily be shown that the eigenvalues verify the dispersion equation (22). In the absence of dissipation, second and fourth eigenvalues are real and are related to attenuating waves. Depending on the sign of $k_{f}^{2}-(q \pi / b)^{2}$, first and third eigenvalues are real or imaginary and are related to effectively propagating waves $\left(k_{f}^{2}-(q \pi / b)^{2}>0\right)$ or evanescent waves $\left(k_{f}^{2}-(q \pi / b)^{2}<0\right)$. The sign of the eigenvalue indicates the travelling direction of the associated 
wave: from the chosen convention first and second eigenvalues are related to travelling waves towards $x<0$ and third and fourth eigenvalues are related to travelling waves towards $x>0$. When several transverse modes are considered, a similar classification is chosen, thus:

$$
\mathbf{\Lambda}=\operatorname{diag}(\mathbf{p}-, \mathbf{a}-, \mathbf{p}+, \mathbf{a}+)
$$

where $\mathbf{p}$ and $\mathbf{a}$ indicate a vector of eigenvalues related to propagating or attenuating waves, respectively, and the sign indicates the direction of travel of the associated waves. The wave vector is thus noted

$$
\mathbf{V}=\left[\begin{array}{c}
\mathbf{V}_{p}^{-} \\
\mathbf{V}_{a}^{-} \\
\mathbf{V}_{p}^{+} \\
\mathbf{V}_{a}^{+}
\end{array}\right]
$$

\subsection{Scattering matrices in the $A B H$ waveguide}

Let $\Omega$ be a region of the waveguide delimited by two abscissas $t_{-}$and $t_{+}$(see Fig. 3). $\quad \mathbf{V}_{\text {out }}$ and $\mathbf{V}_{\text {in }}$ are the outcoming wave vector from $\Omega$ and incoming wave vector to $\Omega$, respectively. They write

$$
\mathbf{V}_{\text {out }}=\left[\begin{array}{c}
\mathbf{V}^{-}\left(t_{-}\right) \\
\mathbf{V}^{+}\left(t_{+}\right)
\end{array}\right] \quad \text { and } \quad \mathbf{V}_{\text {in }}=\left[\begin{array}{c}
\mathbf{V}^{-}\left(t_{+}\right) \\
\mathbf{V}^{+}\left(t_{-}\right)
\end{array}\right]
$$

The scattering matrix $\mathbf{S}($ size $4 K \times 4 K)$ of $\Omega$ is defined by

$$
\mathbf{V}_{\text {out }}=\mathbf{S V}_{\text {in }}
$$

and writes

$$
\mathbf{S}=\left[\begin{array}{cc}
\mathbf{T}^{+-} & \mathbf{R}^{-} \\
\mathbf{R}^{+} & \mathbf{T}^{-+}
\end{array}\right]
$$


where matrix $\mathbf{R}^{+}($size $2 K \times 2 K)$ represents the reflection of the incident waves at side $t_{+}\left(\right.$denoted $\mathbf{V}^{-}\left(t_{+}\right)$) on the reflected waves at side $t_{+}$(denoted $\left.\mathbf{V}^{+}\left(t_{+}\right)\right)$. Matrix $\mathbf{T}^{+-}($size $2 K \times 2 K)$ represents the transmission of the incident waves at side $t_{+}$on the transmitted waves at side $t_{-}$. Moreover, following the form of the wave vector (see Eq. 27), the term $\left(R_{p a}^{+}\right)_{q j}$ quantify the reflection from the incident propagating mode $q$ on the reflected attenuating mode $j$.

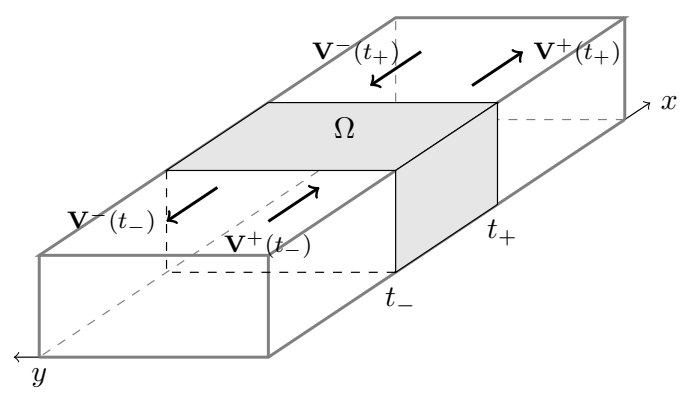

Figure 3: Region $\Omega$ of the waveguide delimited by $x=\left[t_{-}, t_{+}\right]$. Incoming and outgoing waves are represented.

For any region of the waveguide defined by the abscissa $x$ including the end of the waveguide at $x=0$, the scattering matrix $\mathbf{S}_{[0, x]}$ representing the waveguide free extremity is defined in agreement with the definition of Ref. [7]: transmission towards the surrounding medium $\mathbf{T}^{+-}$, transmission towards the inside $\mathbf{T}^{-+}$and reflection of the outside $\mathbf{R}^{-}$are zero. Only $\mathbf{R}^{+}$ is non-zero. Hence:

$$
\mathbf{S}_{[0, x]}=\left[\begin{array}{cc}
\mathbb{O} & \mathbb{O} \\
\mathbf{R}^{+}(x) & \mathbb{O}
\end{array}\right]
$$

Matrix $\mathbf{R}^{+}(x)$ is then called the reflection matrix of the guide extremity (i.e. the region $[0, x])$. Eqs. (17), (20) and (19) yields the relation between $\mathbf{R}^{+}(x)$ 
and $\mathbf{Z}(x)$ :

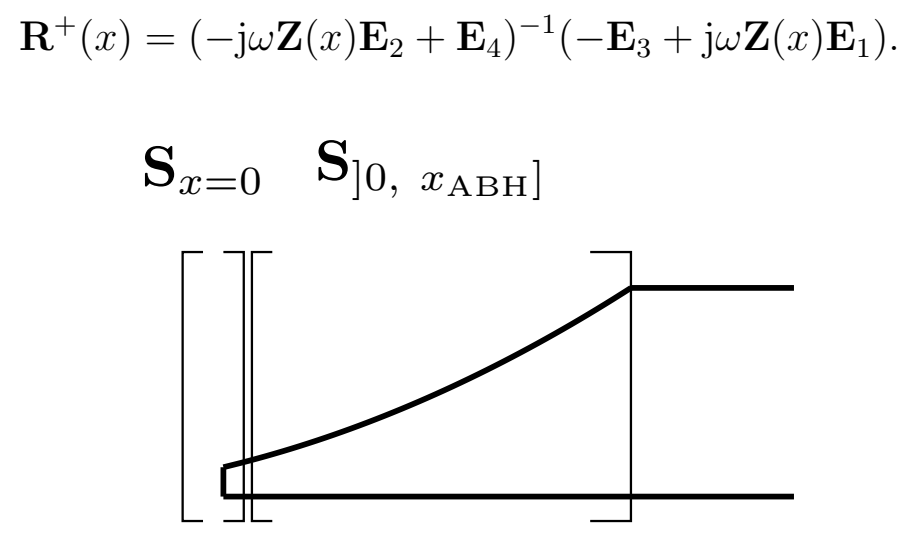

Figure 4: Boundary condition at $x=0$ and region $\left.] 0, x_{\mathrm{ABH}}\right]$. The assembly of the two regions defines region $\left[0, x_{\mathrm{ABH}}\right]$ (see Eq. (33)).

When $x=0, \mathbf{S}_{[0, x]}$ is the scattering matrix of the boundary condition $\mathbf{S}_{x=0}$. The relation between scattering matrix $\mathbf{S}_{[0, x]}$ of region including the boundary condition and scattering matrix $\mathbf{S}_{0, x]}$ of region excluding the boundary condition is (see Fig. 4)

$$
\mathbf{S}_{\left[0, x_{\mathrm{ABH}}\right]}=\mathbf{S}_{x=0} \star \mathbf{S}_{] 0, x_{\mathrm{ABH}}\right]},
$$

where the $\star$ assembly operator is defined in Appendix A. It is therefore important to distinguish $\mathbf{R}^{+}(x)$ and $\left.\left.\mathbf{R}^{+}\right] 0, x\right]$. Practically, Eq. (33) will be used to determine $\mathbf{S}_{\left[0, x_{\mathrm{ABH}}\right]}$. Details about $\mathbf{S}_{x=0}$ and $\mathbf{S}_{00, x]}$ are given in Sec. 2.5 which proposes a model for the imperfect boundary condition, and Sec. 2.6 which proposes to find numerically the scattering in the ABH profile.

\subsection{Imperfect boundary condition}

Identifying and modelling imperfections or defaults in a structure is closely related to structural health monitoring [27] where the main interest is usually 


$$
\begin{gathered}
\rho h a(y) \mathrm{d} y \ddot{z}(y)=-V_{x}(0, y), \\
I_{0} \ddot{\theta}_{x}(0, y)_{x}=-M_{x}(0, y),
\end{gathered}
$$

with $\rho h a(y)$ the mass of the element, assumed concentrated in its centre of mass, whose vertical displacement is $z(y)=w(0, y)+\frac{a(y)}{2} \theta_{x}(0, y)$. The moment of inertia $I_{0}$ writes:

$$
I_{0}=\int_{0}^{a(y)} s^{2} \rho h \mathrm{~d} y \mathrm{~d} s=\frac{\rho h a(y)^{3} \mathrm{~d} y}{3} .
$$


(a)

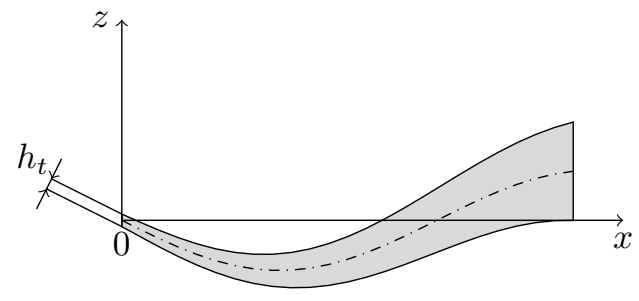

(b)

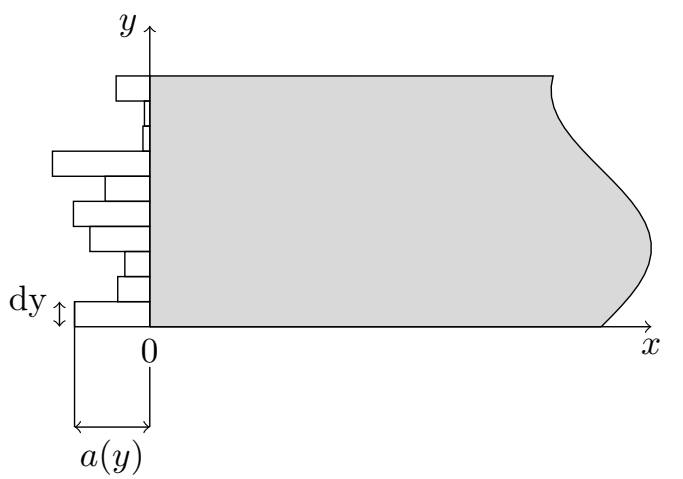

Figure 5: Model of imperfection as an extension constituted of infinitesimal rigid bars: (a) side view and (b) top view. 
Eqs. (34) and (35) then write

$$
\begin{gathered}
V_{x}(0, y)=\rho h a(y) \mathrm{d} y \omega^{2}\left(w+\frac{a \theta_{x}}{2}\right), \\
M_{x}(0, y)=\frac{\rho h a(y)^{3} \mathrm{~d} y}{3} \omega^{2} \theta_{x} .
\end{gathered}
$$

From the impedance matrix definition of Eq. (17), the boundary condition at $x=0$ is expressed as an initial impedance condition:

$$
\mathbf{Z}_{0}=\frac{1}{\mathrm{j} \omega}\left[\begin{array}{cc}
\mathbb{O} & \frac{\rho h \omega^{2}}{3 b} \int_{0}^{b} a(y)^{3} \Psi_{q} \Psi_{j} \mathrm{~d} y \\
\frac{\rho h \omega^{2}}{b} \int_{0}^{b} a(y) \Psi_{q} \Psi_{j} \mathrm{~d} y & \frac{\rho h \omega^{2}}{2 b} \int_{0}^{b} a(y)^{2} \Psi_{q} \Psi_{j} \mathrm{~d} y
\end{array}\right] .
$$

In the general case, matrix $\mathbf{Z}_{0}$ does not have a purely analytical expres215 sion. The reality of imperfections is complex but this model gives a simple way to represent it with a single parameter $a(y)$. This parameter has to be small with regard to the wavelength at $x=0$ for the model to be valid and the bars to respect the assumption of inflexibility. Following this hypothesis, the imperfect termination adds inertial effects but no elastic effects. The elementary academic imperfection presented here induces wave conversion mechanisms similar to those induced by a complex imperfection. The couplings due to elementary imperfections can then be studied.

Because of $a(y)$ is supposed to be small, terms $\frac{\rho h \omega^{2}}{2 b} \int_{0}^{b} a(y)^{2} \Psi_{q} \Psi_{j} \mathrm{~d} y$ and $\frac{\rho h \omega^{2}}{3 b} \int_{0}^{b} a(y)^{3} \Psi_{q} \Psi_{j} \mathrm{~d} y$ can actually be neglected in regards of $\frac{\rho h \omega^{2}}{b} \int_{0}^{b} a(y) \Psi_{q} \Psi_{j} \mathrm{~d} y$.

225 The model of Eq. (39) is then simplified in the followings: the effects of rigid bars are restricted to their localised masses, therefore

$$
\mathbf{Z}_{0}=\frac{1}{\mathrm{j} \omega}\left[\begin{array}{cc}
\mathbb{O} & \mathbb{O} \\
\frac{\omega^{2}}{b} \int_{0}^{b} m(y) \Psi_{q} \Psi_{j} \mathrm{~d} y & \mathbb{O}
\end{array}\right]
$$


with $m(y)=\rho h a(y)$. Computing $S_{x=0}$ is immediate using Eqs. (31), (32) and (40).

\subsection{Numerical resolution for the ABH profile}

\subsubsection{Magnus method}

A Magnus scheme $[29,30,31]$ can be used to solve the Riccati equation (18) in a similar way as in [6]. It is used in this paper for computing the scattering matrix of a section. A fourth-order Magnus scheme is used. The waveguide is discretized along the $x$-direction with a constant step. The Magnus scheme is applied to Eq. (11) and yields:

$$
\mathbf{W}\left(\bar{x}_{n+1}\right)=e^{\boldsymbol{\Omega}_{\mathbf{n}}} \mathbf{W}\left(\bar{x}_{n}\right),
$$

where $\bar{x}_{n}$ is the longitudinal discrete coordinate and $\Omega_{n}$ is the fourth-order Magnus matrix [32]:

$$
\boldsymbol{\Omega}_{\mathbf{n}}=\frac{\Delta}{2}\left(\mathbf{H}_{1}+\mathbf{H}_{2}\right)+\frac{\sqrt{3}}{12} \Delta^{2}\left[\mathbf{H}_{2}, \mathbf{H}_{1}\right],
$$

where $\Delta=\bar{x}_{n+1}-\bar{x}_{n}$ is the constant spatial step,

$$
\begin{aligned}
& \mathbf{H}_{1}=\mathbf{H}\left(\bar{x}_{n}+\left(\frac{1}{2}-\frac{\sqrt{3}}{6}\right) \Delta\right), \\
& \mathbf{H}_{2}=\mathbf{H}\left(\bar{x}_{n}+\left(\frac{1}{2}+\frac{\sqrt{3}}{6}\right) \Delta\right),
\end{aligned}
$$

and $\left[\mathbf{H}_{2}, \mathbf{H}_{1}\right]$ is the commutator between $\mathbf{H}_{2}$ and $\mathbf{H}_{1}$. 


\subsubsection{Computation of the elementary scattering matrix from Magnus scheme} tained from Magnus scheme. Combining Eq. (41) and Eq. (21) gives

$$
\mathbf{V}_{\bar{x}_{n+1}}=\mathbf{E}_{\bar{x}_{n+1}}^{-1} e^{\boldsymbol{\Omega}_{\mathbf{n}}} \mathbf{E}_{\bar{x}_{n}} \mathbf{V}_{\bar{x}_{n}}
$$

The local transfer matrix $\mathbf{Q}[33]$ is identified in Eq. (45):

$$
\mathbf{Q}=\mathbf{E}_{\bar{x}_{n+1}}^{-1} e^{\boldsymbol{\Omega}_{\mathrm{n}}} \mathbf{E}_{\bar{x}_{n}}=\left[\begin{array}{ll}
\mathrm{Q}_{1} & \mathrm{Q}_{2} \\
\mathrm{Q}_{3} & \mathrm{Q}_{4}
\end{array}\right]
$$

Rewriting $\mathbf{V}_{\bar{x}_{n+1}}$ and $\mathbf{V}_{\bar{x}_{n}}$ in $\mathbf{V}_{\text {out }}$ and $\mathbf{V}_{\text {in }}$ (referring to the elementary section) allows to reorganize matrix $\mathbf{Q}$ in order to obtain the scattering matrix $\mathbf{S}_{] 0, x]}$ (in Sec. 3.4) or $\mathbf{S}_{[0, x]}$ (in Sec. 3.2). Namely, if the ABH region is discretized in $N$ elements, it is possible to write its scattering matrix $\mathbf{S}_{\text {region }}$ as

$$
\mathbf{S}_{\text {region }}=\mathbf{S}_{\text {elem }}^{0} \star \mathbf{S}_{\text {elem }}^{1} \star \mathbf{S}_{\text {elem }}^{2} \star \ldots \star \mathbf{S}_{\text {elem }}^{N} .
$$

In the following, the computation is done with $\Delta=10^{-4} \mathrm{~m}$ and 30 modes

have been taken into account. A convergence study (not presented in this paper) shows that using 30 modes for this configuration in the frequency range 
of interest is sufficient to accurately model the effect of the imperfection. The number of modes used should be in any case greater than the number of propagating modes at the $\mathrm{ABH}$ tip at the highest frequency.

260

\section{Scattering induced by imperfections}

\subsection{Characteristics of the simulated waveguide}

The case of an $\mathrm{ABH}$ waveguide made of aluminium is considered. Parameters used in the numerical applications of the model are summarized in Tab. 1; parameters for the damping layer are realistic but do not result from characterization tests. Moreover, as a first step the imperfection is modelled by a single mass localised at $\left(x=0, y_{0}=b / 2\right)$. The mass represents about $0.2 \%$ of the total mass of the $\mathrm{ABH}$ termination. This mass localisation induces that only waveguide modes with odd numbers are concerned with possible coupling. The initial condition (40) then simplifies:

$$
\mathbf{Z}_{0}=-\frac{\mathrm{j} m_{0} \omega}{b}\left[\begin{array}{cc}
\mathbb{O} & \mathbb{O} \\
\Psi_{j}\left(y_{0}\right) \Psi_{k}\left(y_{0}\right) & \mathbb{O}
\end{array}\right]
$$

In this configuration, mode 1 is propagating above $360 \mathrm{~Hz}$ in the homogeneous region (this is assessed further in Sec. 3.3) and the next odd mode is mode 3 which is propagating above $2000 \mathrm{~Hz}$. Therefore, numerical results concerning mode 1 are shown in the 400-2000 Hz frequency range.

\subsection{Typical results for the reflection coefficient}

Fig. 6 presents variations of reflection coefficient $\left(R_{p p}^{+}\right)_{11}$ (of incident mode 1 on reflected mode 1) with frequency, for terminations described in Tab. 1 


\begin{tabular}{lc}
\hline Geometrical characteristics & Characteristics of material \\
\hline \multicolumn{2}{c}{ Aluminium plate } \\
$L=0.8 \mathrm{~m}, b=0.1 \mathrm{~m}, \quad E_{1}=70 \mathrm{GPa}, \eta_{1}=0.2 \%$, \\
$x_{\mathrm{ABH}}=0.06 \mathrm{~m}, x_{0}=0.01 \mathrm{~m}, \quad \rho_{1}=2700 \mathrm{~kg} \cdot \mathrm{m}^{-3}, \nu=0.3$. \\
$h_{0}=1.5 \mathrm{~mm}, m=2$. \\
$\quad$ Damping layer \\
$h_{l}=0.1 \mathrm{~mm}, \quad E_{2}=7 \mathrm{GPa}, \eta_{2}=20 \%$, \\
$x_{l}=0.05 \mathrm{~m}$. \\
$\rho_{2}=1000 \mathrm{~kg} \cdot \mathrm{m}^{-3}$. \\
$y_{0}=0.05 \mathrm{~m} \quad$ Imperfection \\
\hline
\end{tabular}

Table 1: Geometrical and material characteristics of the simulated ABH waveguide.

with or without imperfection. The reflection on the ABH extremity without imperfection and without damping layer (full gray line) is close to unity. This is not surprising since the non covered ABH is known to be inefficient [8]. Covered $\mathrm{ABH}$ without imperfection (dashed gray line) gives an interesting result as its reflection coefficient can be as low as 0.5 with oscillations; this results can be compared to earlier results [6].

The case of the non covered but imperfect ABH termination (single mass at the centre of the cross-section, full black line) is mostly identical to the perfect termination except that it displays several very deep minima localised in frequency. With a damping layer the imperfect termination (dashed black line), $\left|\left(R_{p p}^{+}\right)_{11}\right|$ clearly leads to a smaller reflection than the perfect covered case; the difference can be as much as 0.1. Notice that local minima seen on the non-covered imperfect extremity can still be observed in the covered case but are much less localised in frequency. 


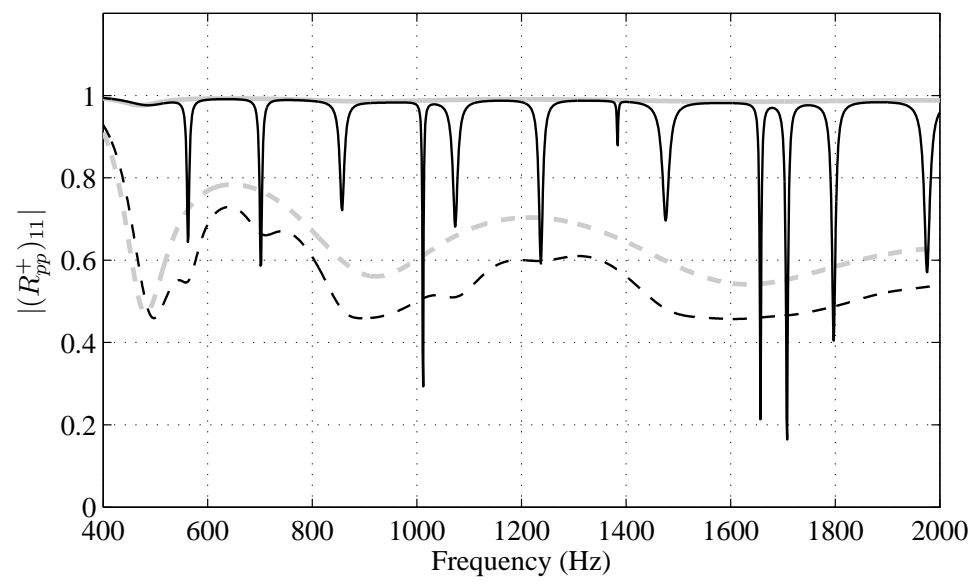

Figure 6: Modulus of the reflection coefficient $\left(R_{p p}^{+}\right)_{11}$ for perfect non covered (full gray line), perfect covered (dashed gray line), imperfect non covered (full black line) and imperfect covered (full dashed line) ABH terminations (parameters of Tab. 1).

\subsection{Cut-off frequency}

In an homogeneous structure of a given thickness, whether a mode $q$ is propagating or not only depends on the frequency according to the dispersion relation for mode $q$ (see Eq. (22)). In an inhomogeneous structure such as the tapered profile studied here (see Fig. 7(a)), the propagating behaviour depends on both the frequency and the thickness, therefore on the axial coordinate.

At a given frequency, it is shown on Fig. 7(b) that the eigenvalues associated with propagating waves of modes $q=1,3,5,7$ (for example) are functions of abscissa (see Eq.(22)). A mode becomes propagating at its cutoff abscissa, which is indicated by the change of nature of the associated eigenvalue (real or imaginary). These cut-off abscissas allow for each mode $q$ to define coloured region on Fig. 7(a). Fig. 7(c) shows that the cut-off 
frequency of mode $q$ depends on the abscissa, i.e. on the local thickness of is propagating above $360 \mathrm{~Hz}$ while modes 3,5 and 7 are not. They are thus confined in the tapered extremity.

\subsection{Reflection induced by the tapered profile itself}

The geometrical acoustics analysis [8], which is based on the WKB approximation and only considers propagating waves, suggests that an incident wave reflects only at the boundary and that there is no reflection inside the tapered profile. The model presented in Sec. 2 gives the scattering properties in a region $\left.] 0, x_{\mathrm{ABH}}\right]$ of the waveguide that does not include the free boundary condition. Zero reflection implies that the scattering matrix is diagonal: ing matrices $\mathbf{S}_{] 0, x]}$ and $\mathbf{S}_{[0, x]}$ are computed with respect to frequency and allow to observe the reflection of the $\mathrm{ABH}$ profile with or without the free condition, respectively.

Fig. 8 shows indeed that for mode 1 , there is a non zero reflection along the tapered section reflects a low part of incident waves (full gray line). Note that covering the profile with a damping layer does not affect this behaviour consistently (full black line). The observed reflection coefficient displays oscillations that can directly be compared to oscillations in the full ABH termination (i.e. including the boundary condition, dashed black line). Oscillations of the reflection coefficient have been observed first in [6], where the authors hypothesize that oscillations are due to sharpness and length of 


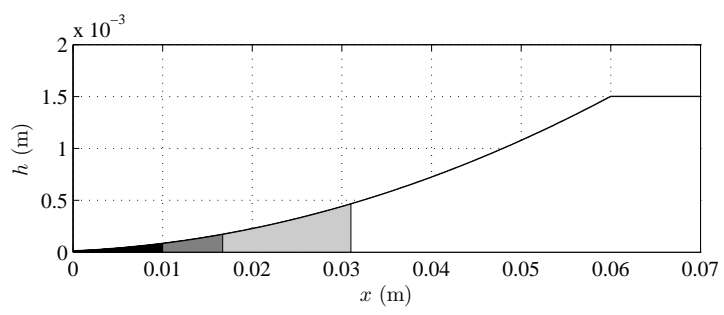

(a)

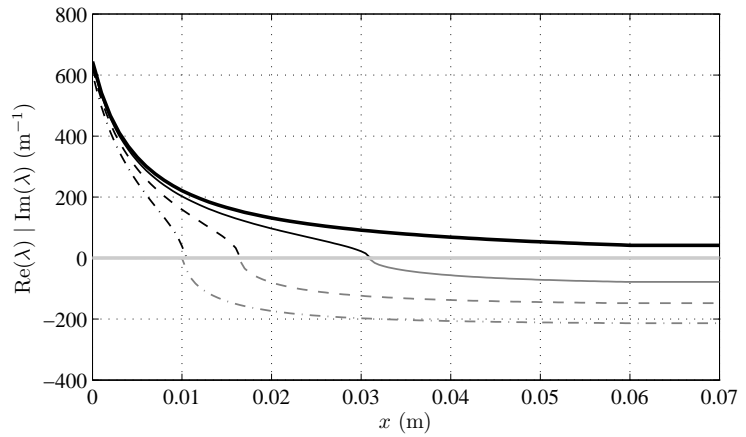

(b)

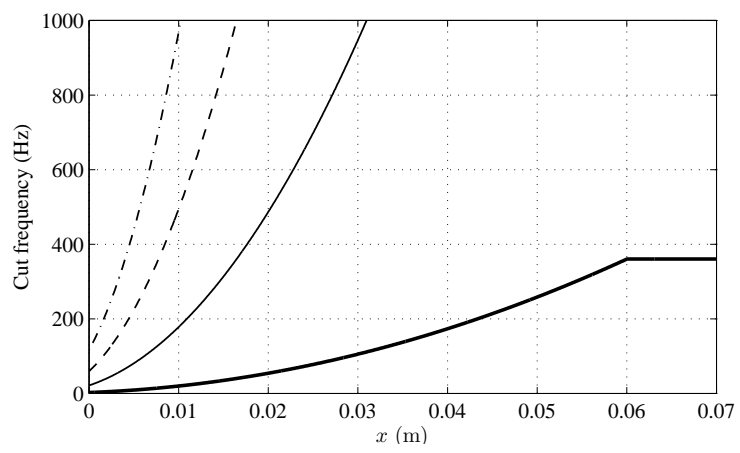

(c)

Figure 7: (a) Thickness profile of the extremity with zone of propagation of modes $k<3$ (white), $k<5$ (light gray), $k<7$ (dark gray) and $k>7$ (black) at $1000 \mathrm{~Hz}$. (b) Real (grey) and imaginary (black) parts of $\lambda_{1}$ (thick full), $\lambda_{3}$ (thin full), $\lambda_{5}$ (dashed) and $\lambda_{7}$ (dashdotted) at $1000 \mathrm{~Hz}$. (c) Cut-off frequency for mode 1 (thick full), 3 (thin full), 5 (dashed), 7 (dashdotted). 
the profile. Note that the geometrical acoustics approach and the associated WKB solution $[8,34]$ does not yield oscillations; therefore, it may indicate that the approximations made in this approach are not valid for the studied geometry, especially the approximation of sufficient smoothness. This is also recently suggested in a study of Feurtado et al. [35] who study the parameters yielding a smooth profile, but was also briefly discussed in Ref. [34].

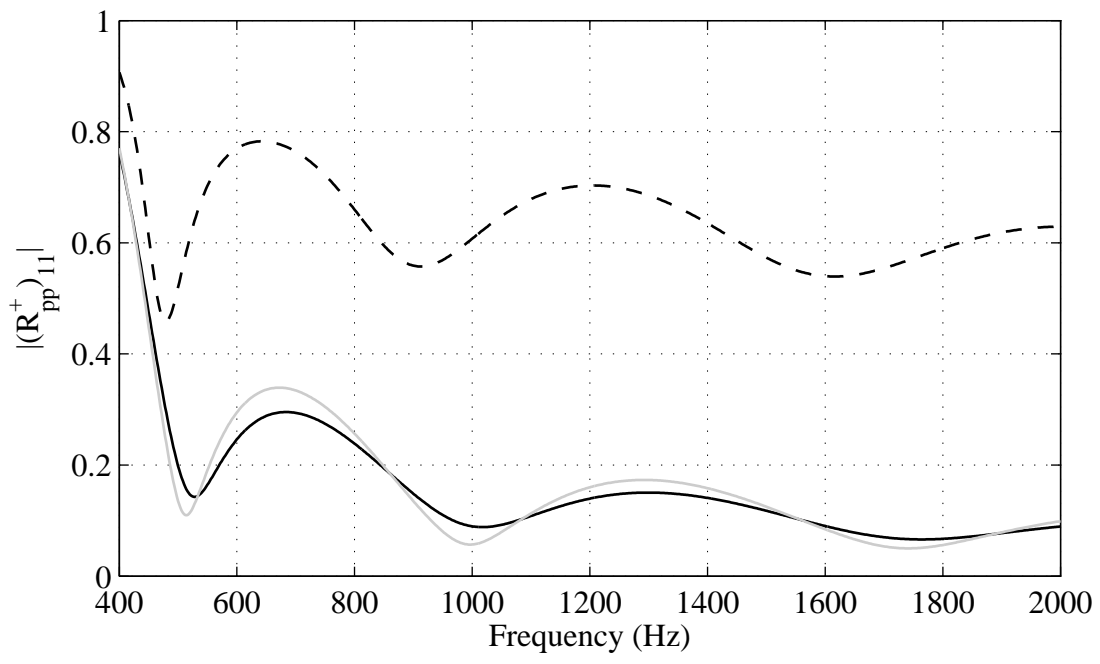

Figure 8: Modulus of the term $(1,1)$ of the reflection matrix $\left.\mathbf{R}_{j 0}^{+}, x_{\mathrm{ABH}}\right]$ with damping layer (full black), without damping layer (full grey) and of the reflection matrix $\mathbf{R}^{+}(x)$ with damping layer (dashed black).

\subsection{Coupling mechanisms and mode trapping}

Let us consider a propagating incident wave $q$ arriving at the extremity. The reflection matrix $\mathbf{R}_{p p}^{+}(x=0)$ is displayed on Fig. 9(a). It shows the mode coupling at the imperfect free end: the diagonal terms of $\left|\mathbf{R}_{p p}^{+}\right|$represent the reflection of a mode on itself, and are less that unity in this case. The out-ofdiagonal terms represent the reflection of an incident mode on one or several 

is not covered with a damping layer. It suggests that only at these frequencies the energy is trapped and that there exist local resonances responsible for the drop in the reflection coefficient. 


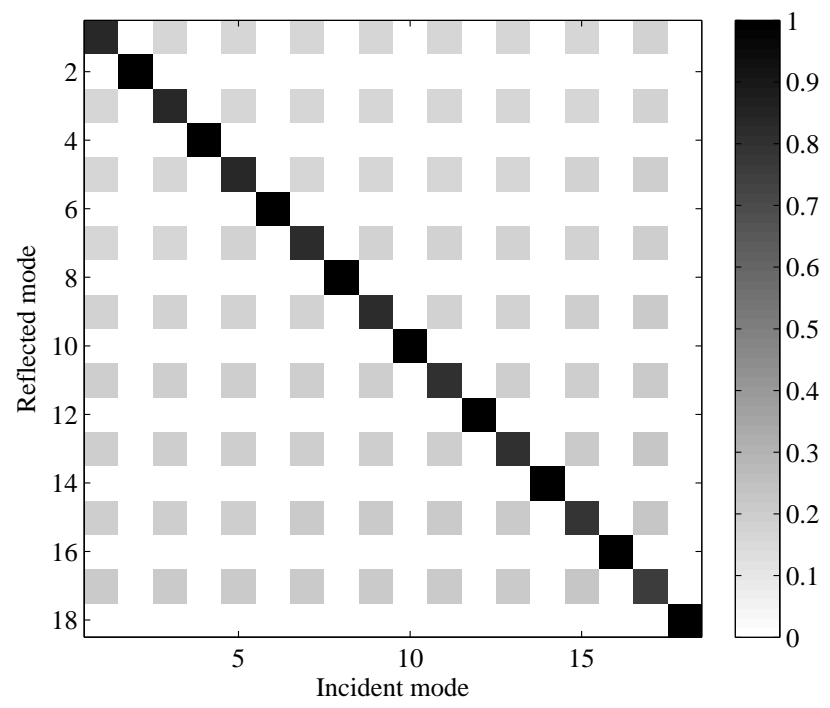

(a)

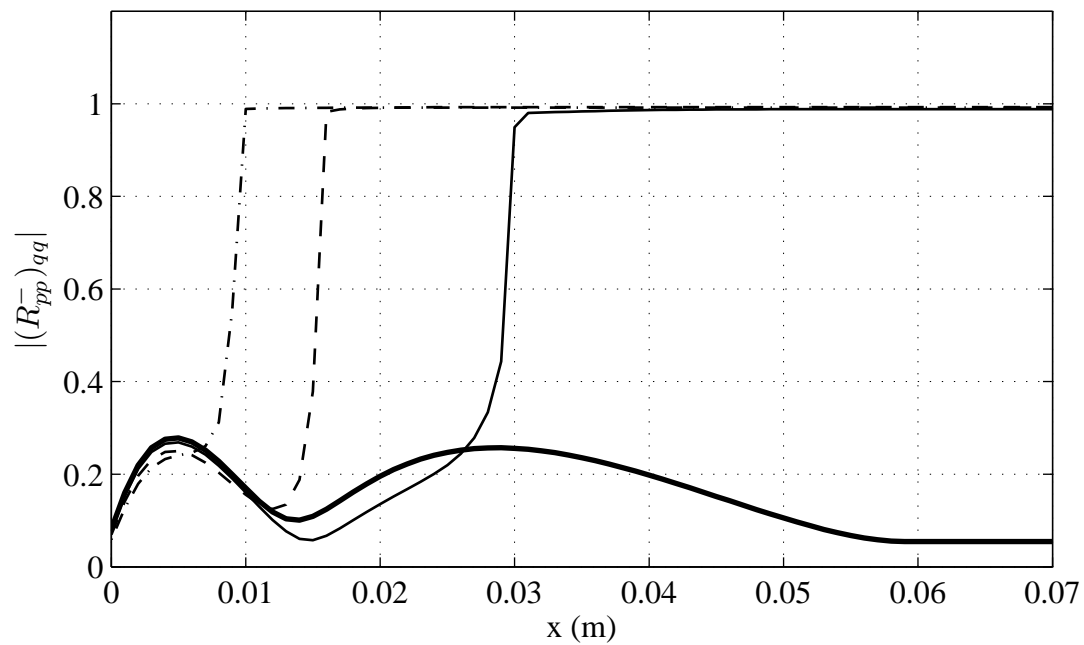

(b)

Figure 9: (a) Reflection matrix $\left|\mathbf{R}_{p p}^{+}\right|$at point $x=0$ and for $f=1000 \mathrm{~Hz}$ and (b) Third (full), fifth (dashed) and seventh (dashdotted) diagonal terms of the reflection matrix $\left|\mathbf{R}_{p p}^{-}\right|$ of the region $] 0, x]$ at $f=1000 \mathrm{~Hz}$. 


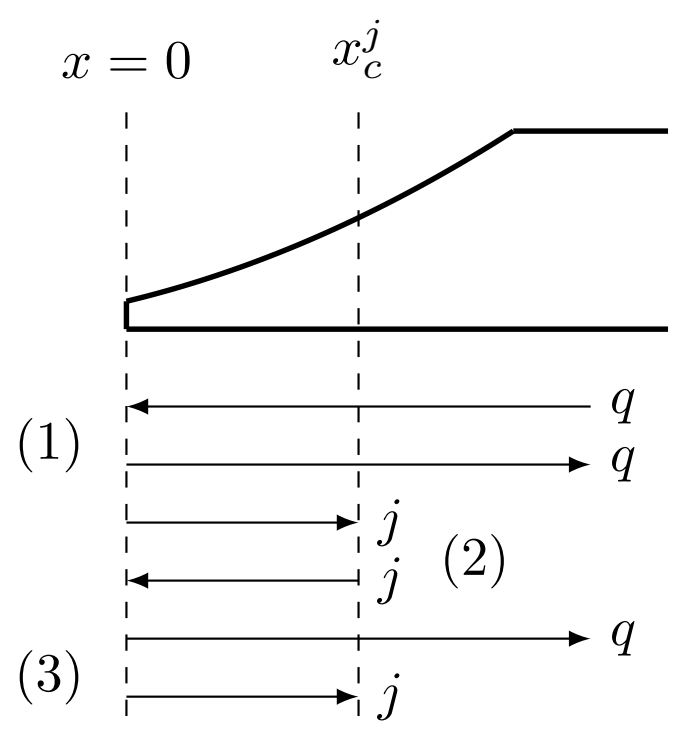

Figure 10: Scheme of the excitation and reflection mechanisms in the imperfect $A B H$ termination. $x_{c}^{j}$ indicates the cut-off abscissa for mode $j$. In step (1) the incident mode $q$ is scattered on mode $j$, in step (2) mode $j$ is reflected towards the extremity and in step (3) mode $j$ is scattered on mode $q$. 


\subsubsection{Effect of the position of the imperfection}

Let us consider the same mass figuring the imperfection at two more positions: $y_{0}=b / 3$ and $y_{0}=4 b / 5$. Reflection matrices for these cases are plotted on Fig. 11 and show the couplings between modes; note the slight differences compared to Fig. 9(a). Fig. 12 shows the results for $\left|\left(R_{p p}^{+}\right)_{11}\right|$ in these configurations. In the case of the non covered tapered profile, reflection coefficients for $b / 2$ and $b / 3$ are different as they do not display the same minima: this can be expected since they do not couple the same modes. When the termination is covered, the three cases are rather similar (see Fig. 13). It is however noticeable, that the best performance is reached when $y_{0}=b / 2$, which is the position of maximum displacement for mode 1.

\subsubsection{Effect of the number of imperfections}

Fig. 13 presents results on $\left|\left(R_{p p}^{+}\right)_{11}\right|$ for combined imperfections. Combination $b / 2+b / 3$ and $b / 2+b / 3+4 b / 5$ are studied. Note that theses configurations 380 combines the minima of cases studied in Sec. 3.6.1. Cancellation of mutual effect may appear at some frequencies when the termination is not covered. Broad drops of $\left|\left(R_{p p}^{+}\right)_{11}\right|$ appears (at $1600 \mathrm{~Hz}$ ) that are the consequence of two close consecutive minima. In the covered case, combining imperfections seems beneficial for the reflection coefficient which is reduced in the 500-700, 385

900-1100 and 1400-2000 Hz ranges when masses are added to the model. The gain reaches 0.2 at some frequencies. Compared to Sec. 3.6.1, it appears that multiplying the imperfections helps obtaining a significant reduction 


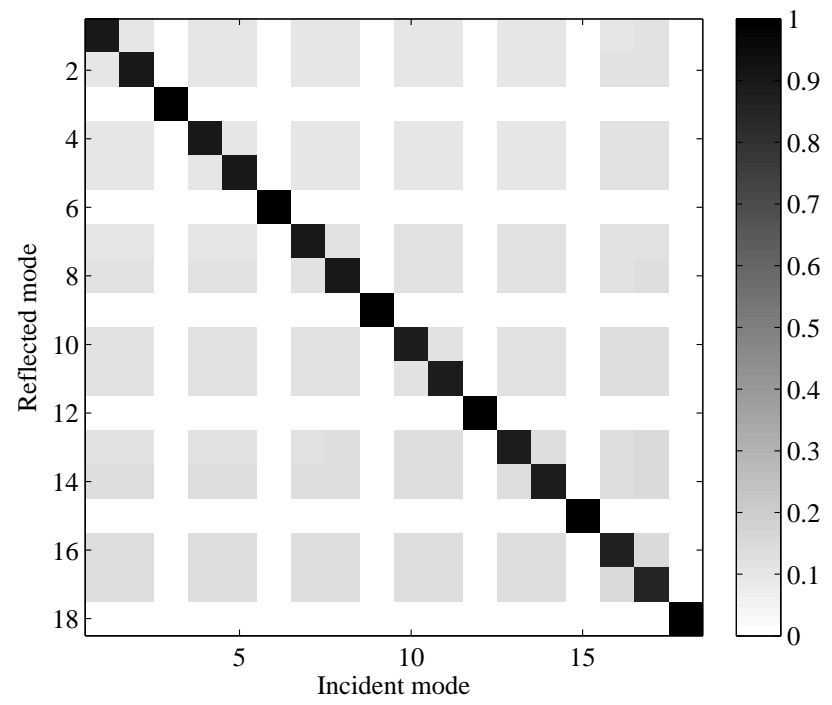

(a)

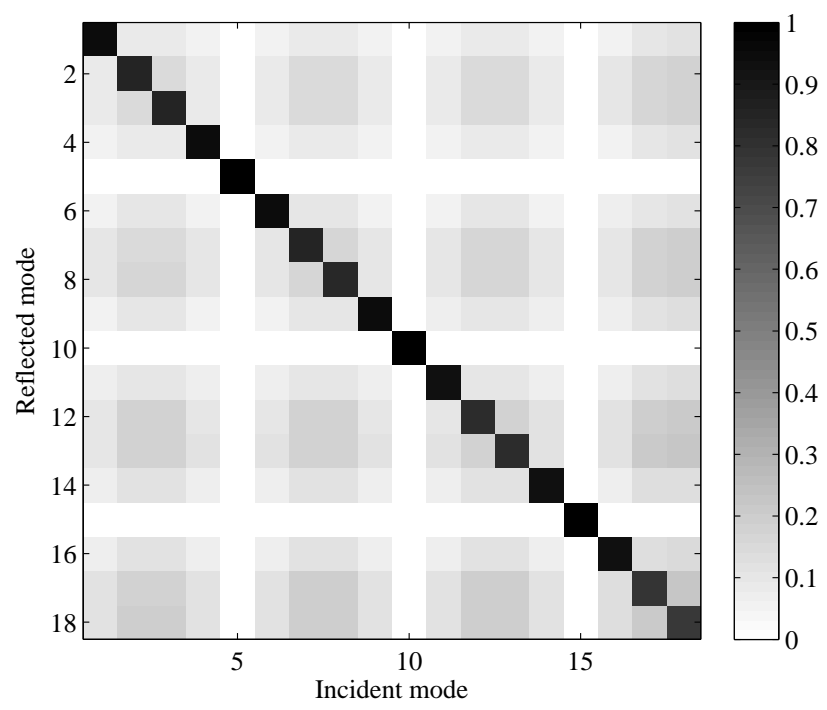

(b)

Figure 11: Reflection matrices $\left|\mathbf{R}_{p p}^{+}\right|$at $x=0,1000 \mathrm{~Hz}$ for masses located in (a) $y=b / 3$ and (b) $y=4 b / 5$. 


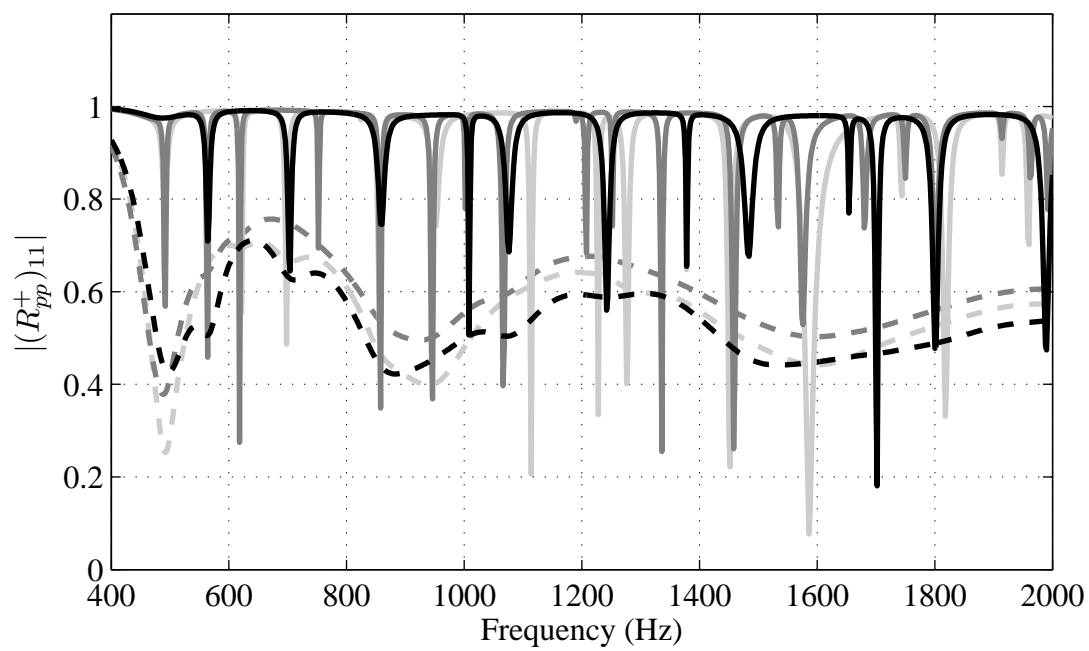

Figure 12: Modulus of the reflection coefficient $\left(R_{p p}^{+}\right)_{11}$ for imperfect cases of ABH termination with mass at $y=b / 2$ (black), $y=b / 3$ (light grey) and $y=4 b / 5$ (dark grey), non covered (full lines) and covered (dashed lines).

of $\left|\left(R_{p p}^{+}\right)_{11}\right|$, assuming that there is no cancellation effects. This fact suggests that naturally obtained imperfections are not necessarily detrimental and that controlled irregularities of the $\mathrm{ABH}$ extremity can be used in order to enhance its damping performance for a given minimum thickness; it is reminded that a thinner extremity provides better results.

\section{Experimental investigations on an imperfect $\mathrm{ABH}$ termination}

\subsection{Experimental setup}

This experiment uses the setup and the method detailed by the authors in [19] for measuring the reflection coefficient of a beam. The method is similar to Kundt-method in acoustics. A wave model and a least-square technique 


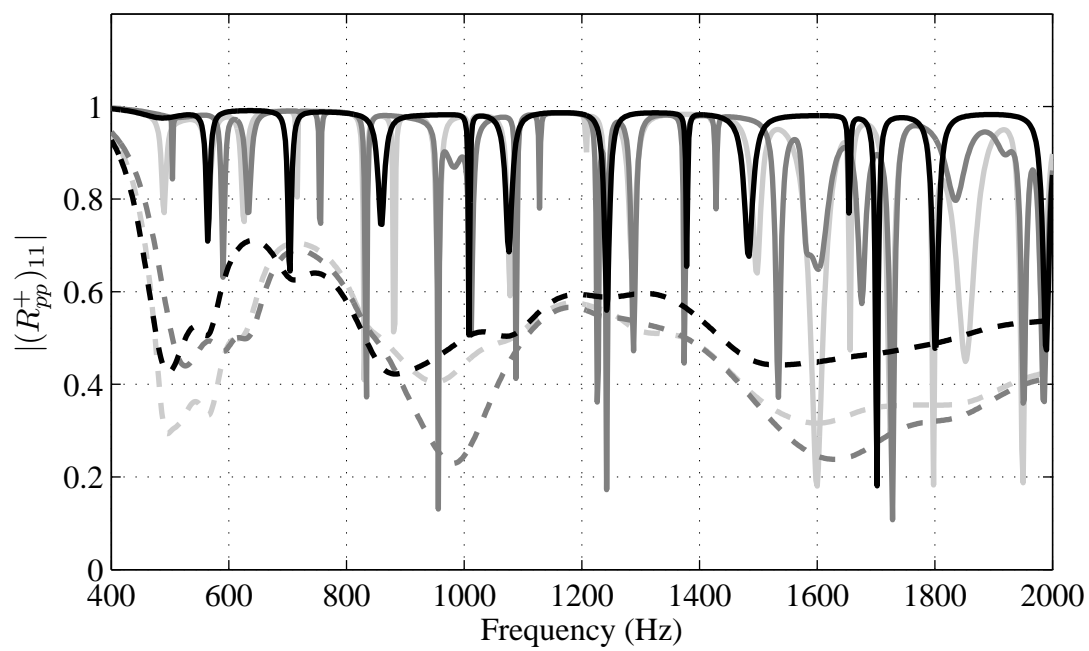

Figure 13: Modulus of the reflection coefficient $\left(R_{p p}^{+}\right)_{11}$ for imperfect cases of ABH termination with mass at $y=b / 2$ (black), $y=[b / 2 ; b / 3]$ (light grey) and $y=[b / 2 ; b / 3 ; 4 b / 5]$ (dark grey), non covered (full lines) and covered (dashed lines).

are used to get the reflection coefficient from frequency response functions at several locations on the beam. The tested beam is vertically suspended and is excited by a swept-sine from 100 to $8000 \mathrm{~Hz}$ using a shaker. Beam velocity is measured using a laser vibrometer (Polytec OFV-400) at 21 abscissas in a $0.1 \mathrm{~m}$ long measurement zone.

The unique sample consists in an aluminium beam with an ABH extremity (see Fig. 14(a)), whose parameters are described in Tab. 2. The tip is as clean and undamaged as possible but displays a small permanent deformation (less than a millimeter) and a small notch. A thin damping layer (striped tape) is stuck on the flat side of the tapered profile (see Fig. 14(b)). This configuration is referred as "non-damaged". With the help of a FEM model (COMSOL) of this beam, the first transverse ABH mode can be found at 1050 

point mass (tin drop) is stuck at the tip of the tapered profile (see Fig. 14(c)). This second configuration is referred as "damaged". A second measurement of the reflection coefficient is then made on the damaged beam.

\begin{tabular}{cc}
\hline Geometrical characteristics & Characteristics of material \\
\hline \multicolumn{2}{c}{ Aluminium beam } \\
$L=0.8 \mathrm{~m}, b=0.02 \mathrm{~m}, \quad E_{1}=70 \mathrm{GPa}, \eta_{1}=0.2 \%$, \\
$x_{\mathrm{ABH}}=0.06 \mathrm{~m}, x_{0}=0.01 \mathrm{~m}, \quad \rho_{1}=2700 \mathrm{~kg} \cdot \mathrm{m}^{-3}$. \\
$h_{0}=1.5 \mathrm{~mm}, m=2$. \\
Imperfection $($ tin drop) \\
$y_{0}=0.01 \mathrm{~m} \quad m_{0}=3.6 \times 10^{-4} \mathrm{~kg}$ \\
\hline
\end{tabular}

Table 2: Geometrical and material characteristics of the sample ABH.

\subsection{Reflection coefficient of an imperfect ABH extremity}

The resulting reflection coefficient for the two configurations can be seen in Fig. 15. On the one hand the reflection coefficient for the non damaged configuration (gray curve) decreases with oscillations below $4000 \mathrm{~Hz}$ to reach very low values (0.1 in magnitude) above $4000 \mathrm{~Hz}$. This behaviour, including the oscillatory phenomenon, is conform to the $\mathrm{ABH}$ models developed in the literature $[4,6,19]$. On the other hand, the damaged configuration (black curve) displays a similar behaviour but presents lower values in the 0-4000 $\mathrm{Hz}$ region. Above $4000 \mathrm{~Hz}$, there is no sensible difference between the two configurations. The differences in magnitude can reach 0.2 and are due to the addition of the point mass at the $\mathrm{ABH}$ tip. This experimental result 


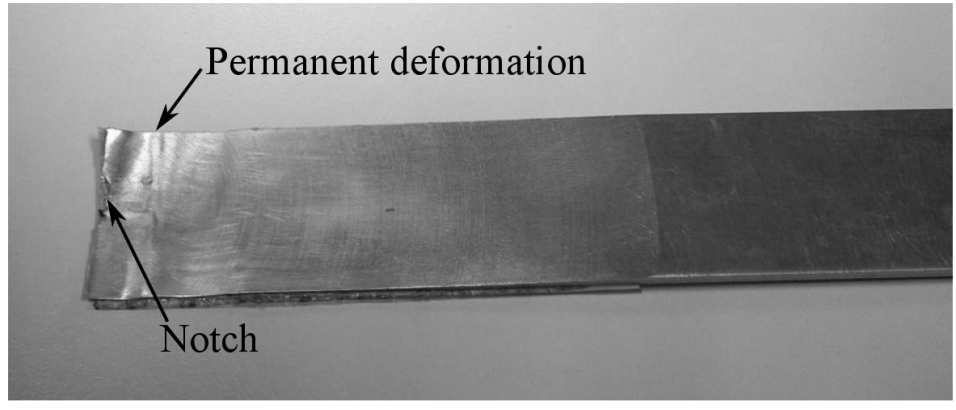

(a)

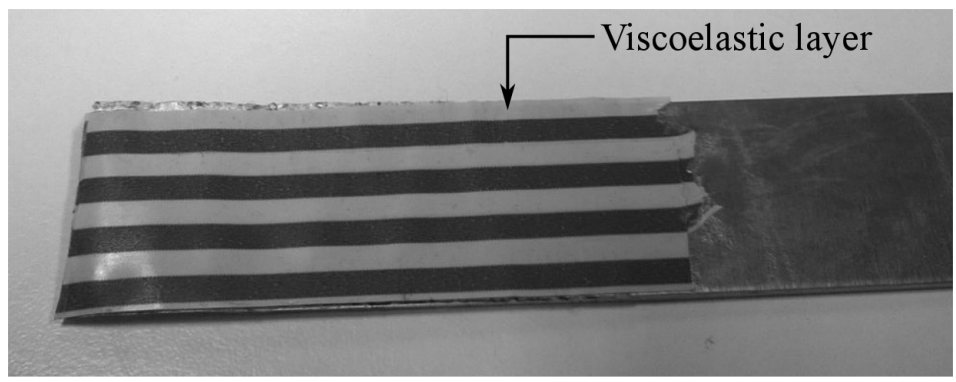

(b)

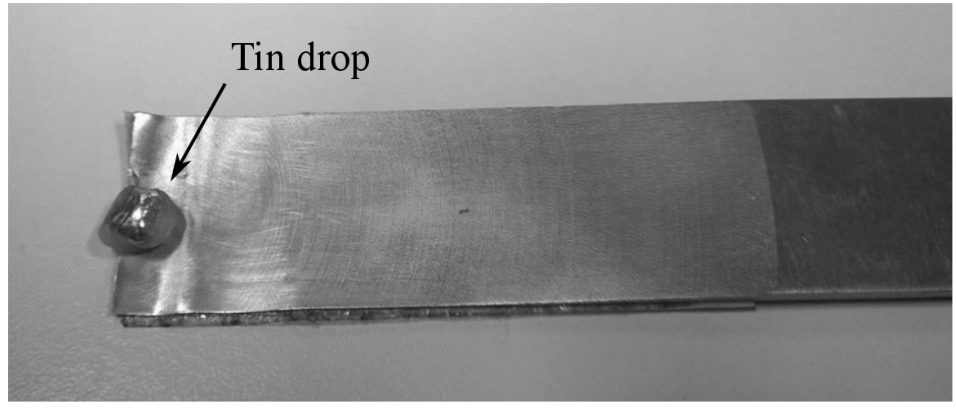

(c)

Figure 14: (a) Machined side of the tapered profile, (b) flat side of the tapered profile, covered with viscoelastic layer (striped tape) and (c) extremity with stuck tin drop. 


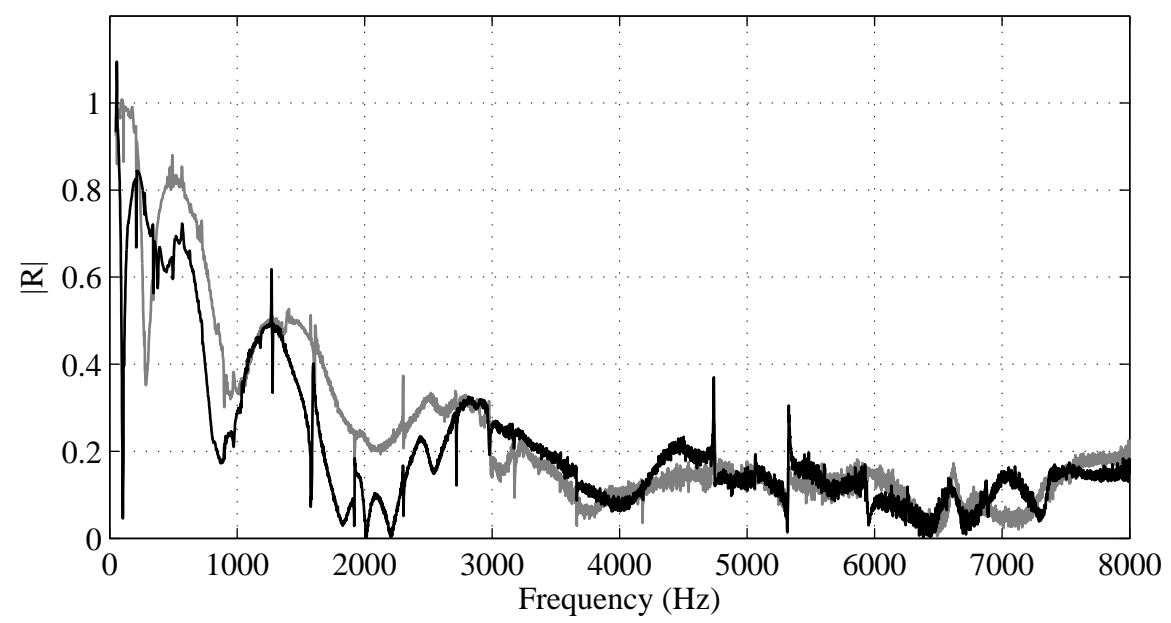

Figure 15: Modulus of the reflection coefficient for the "non damaged" (gray line) and "damaged" (black line) ABH termination

\section{Conclusions}

This paper investigates the role of imperfections that are usually found at the tip of the $\mathrm{ABH}$ tapered profile when it is made very thin, due to the limitations of the manufacturing techniques.

A multimodal model of inhomogeneous waveguide with an ABH termination with a simple imperfection is developed in order to obtain the scattering matrix of a region of the waveguide as well as the reflection matrix of the $\mathrm{ABH}$ extremity. It is numerically solved using a Magnus scheme. It is shown that an elementary imperfection on the free extremity of the tapered profile affects the reflection coefficient and reduces it. Effects are spectrally localised when the damping is weak, but are broadly extended when the damping is increased due to the viscoelastic layer. At a given frequency, guided modes can 
be propagating in the tapered region while they are attenuating in the thicker uniform region of the waveguide. They are thus confined or localised in the tapered region. Due to couplings at the extremity, an incident waveguide mode is partially reflected on multiple modes. Because of the imperfection, guided modes that are confined in the $\mathrm{ABH}$ are excited, leaving the possibility of local resonances that can explain the drop of the reflection coefficient for the incident mode. Furthermore, the results suggest the use of controlled imperfection of the tip of the $\mathrm{ABH}$ profile in order to enhance its damping performance. A key point for researchers and designers of $\mathrm{ABH}$ systems is that imperfect extremities are not detrimental and can at best be beneficial.

Moreover, it is also shown that waves may be reflected inside the tapered profile itself and not only by the extremity. It suggests that many experimental ABH profiles do not match the sufficient smoothness conditions described in [8] and can be improved practically to avoid internal reflections.

Finally, a measurement of the reflection coefficient of an artificially damaged $\mathrm{ABH}$ extremity is realised and shows that it differs from the reflection coefficient of a non damaged extremity. It is used to qualitatively confirm the results of the model.

\section{Appendix A. Assembly rule for scattering matrices}

In order to compute the scattering matrix of a whole inhomogeneous guide from scattering matrices of elementary regions, it is useful to define an operator that combine the scattering matrices $\mathbf{S}_{A}$ and $\mathbf{S}_{B}$ of two consecutive regions $A$ and $B$. If $\mathbf{S}_{A B}$ is the scattering matrix of the concatenation of the 
two regions, it can be shown that $\mathbf{S}_{A B}$ can be computed from $\mathbf{S}_{A}$ and $\mathbf{S}_{B}$ by using a $\star$ operator $[7]$ :

$$
\mathbf{S}_{A B}=\mathbf{S}_{A} \star \mathbf{S}_{B} .
$$

with

$\mathbf{S}_{A B}=\left[\begin{array}{cc}\mathbf{T}_{A B}^{+-} & \mathbf{R}_{A B}^{-} \\ \mathbf{R}_{A B}^{+} & \mathbf{T}_{A B}^{-+}\end{array}\right], \quad \mathbf{S}_{A}=\left[\begin{array}{cc}\mathbf{T}_{A}^{+-} & \mathbf{R}_{A}^{-} \\ \mathbf{R}_{A}^{+} & \mathbf{T}_{A}^{-+}\end{array}\right], \quad \mathbf{S}_{B}=\left[\begin{array}{cc}\mathbf{T}_{B}^{+-} & \mathbf{R}_{B}^{-} \\ \mathbf{R}_{B}^{+} & \mathbf{T}_{B}^{-+}\end{array}\right]$.

Eq. (A.1) is also called assembly rule for scattering matrices. The four submatrices $\mathbf{T}_{A B}^{+-}, \mathbf{R}_{A B}^{-}, \mathbf{R}_{A B}^{+}$and $\mathbf{T}_{A B}^{-+}$of $\mathbf{S}_{A B}$ write

$$
\begin{gathered}
\mathbf{T}_{A B}^{+-}=\mathbf{T}_{A}^{+-}\left(\mathbf{I}-\mathbf{R}_{B}^{-} \mathbf{R}_{A}^{+}\right)^{-1} \mathbf{T}_{B}^{+-}, \\
\mathbf{R}_{A B}^{-}=\mathbf{R}_{A}^{-}+\mathbf{T}_{A}^{+-}\left(\mathbf{I}-\mathbf{R}_{B}^{-} \mathbf{R}_{A}^{+}\right)^{-1} \mathbf{R}_{B}^{-} \mathbf{T}_{A}^{-+}, \\
\mathbf{R}_{A B}^{+}=\mathbf{R}_{B}^{+}+\mathbf{T}_{B}^{-+}\left(\mathbf{I}-\mathbf{R}_{A}^{+} \mathbf{R}_{B}^{-}\right)^{-1} \mathbf{R}_{A}^{+} \mathbf{T}_{B}^{+-}, \\
\mathbf{T}_{A B}^{-+}=\mathbf{T}_{B}^{-+}\left(\mathbf{I}-\mathbf{R}_{A}^{+} \mathbf{R}_{B}^{-}\right)^{-1} \mathbf{T}_{A}^{-+} .
\end{gathered}
$$




\section{References}

${ }_{465}^{6}$ [1] M. D. Rao, Recent applications of viscoelastic damping for noise control in automobiles and commercial airplanes, Journal of Sound and Vibration 262 (2003) 457-474.

[2] L. Cremer, M. Heckl, Structure-Borne Sound: structural vibrations and sound radiation at audio frequencies, second edition, Springer, 1988.

[3] D.I.G. Jones, W.J. Trapp, Influence of additive damping on resonance fatigue of structures, Journal of Sound and Vibration 17 (2) (1971) 157185.

[4] V. V. Krylov, New type of vibration dampers utilising the effect of acoustic 'black holes', Acta Acustica United with Acustica 90 (2004) 830-837.

[5] V.V. Krylov, R.E.T.B. Winward, Experimental investigation of the acoustic black hole effect for flexural waves in tapered plates, Journal of Sound and Vibration 300 (1-2) (2007) 43-49.

[6] V.B. Georgiev, J. Cuenca, F. Gautier, L. Simon, V.V. Krylov, Damping of structural vibrations in beams and elliptical plates using the acoustic black hole effect, Journal of Sound and Vibration 330 (11) (2011) 24972508.

[7] C. Vemula, A.N. Norris, G.D. Cody, Attenuation of waves in plates and bars using a graded impedance interface at edges, Journal of Sound and Vibration 196 (1) (1996) 107-127. 
[8] M.A. Mironov, Propagation of a flexural wave in a plate whose thickness decreases smoothly to zero in a finite interval, Soviet Physics: Acoustics 34 (3) (1988) 318-319.

[9] V.B. Georgiev, J. Cuenca, F. Gautier, M. Moleron, L. Simon, Numerical and experimental investigation of the acoutic black hole effect for vibration damping in beams and elliptical plates, in: Euronoise 2009, Edinburgh, 2009.

[10] V. Denis, A. Pelat, F. Gautier, B. Elie, Modal overlap factor of a beam with an acoustic black hole termination, Journal of Sound and Vibration 333 (2014) 2475-2488.

[11] F. Gautier, J. Cuenca, V.V. Krylov, L. Simon, Experimental investigation of the acoustic black hole effect for vibration damping in elliptical plates, in: Acoustic'08, Paris, 2008.

[12] E.P. Bowyer, D.J. O’Boy, V.V. Krylov, J.L. Horner, Effect of geometrical and material imperfections on damping flexural vibrations in plates with attached wedges of power law profile, Applied Acoustics 73 (5) (2012) 514-523.

[13] E.P. Bowyer, D.J. O’Boy, V.V. Krylov, F. Gautier, Experimental investigations of damping flexural vibrations in plates containing tapered indentations of power-law profile, Applied Acoustics 74 (2013) 553-560.

[14] D.J. O'Boy, V.V. Krylov, Damping of flexural vibrations in circular plates with tapered central holes, Journal of Sound and Vibration 330 (10) (2011) 2220-2236. 
[15] O. Aklouche, A. Pelat, S. Maugeais, F. Gautier, Scattering of flexural

[21] A. Leissa, Vibration of plates, Acoustical society of America, London, 1993.

[22] D.J. Gorman, A general solution for the free vibration of rectangular plates resting on uniform elastic edge supports, Journal of Sound and

[16] O. Aklouche, A. Pelat, S. Maugeais, F. Gautier, Model of the scattering of flexural waves from a two-dimensional acoustic black hole, in: Congrès Français d'Acoustique, Poitiers, 22-25 April 2014, 2014.

[17] V. Denis, Vibration damping in beams using the acoustic black hole effect, Ph.D. thesis, Université du Maine (2014).

[18] V. Denis, J. Poittevin, A. Pelat, F. Gautier, P. Picart, C. Pézerat, Characteristics of the vibration field inside an acoustic black hole placed on a beam, in: ICEDyn 2015, Lagos, Portugal, 22-24 June 2015, 2015.

[19] V. Denis, F. Gautier, A. Pelat, J. Poittevin, Measurement and modelling of the reflection coefficient of an acoustic black hole termination, Journal of Sound and Vibration 349 (2015) 67-79.

[20] D. Ross, E.L. Ungar, E.M. Kerwin, Damping of plate flexural vibrations by means of viscoelastic laminae, in: Structural damping, Pergamon Press Edition, J.E. Ruzicka, Oxford, 1960, pp. 49-57. Vibration 139 (1990) 325-335. 
[23] S.Y. Lee, S.M. Lin, Levy-type solution for the analysis of nonuniform plates, Computers and Structures 49 (6) (1993) 931-939.

[24] Y. Xiang, Y.B. Zhao, G.W. Wei, Levy solutions for vibration of multispan rectangular plates, International Journal of Mechanical Sciences 44 (2002) 1195-1218.

[25] J.-L. Guyader, Vibration in Continuous Media, ISTE Ltd, London, 2006.

[26] S. Felix, V. Pagneux, Multimodal analysis of acoustic propagation in three-dimensional bends, Wave Motion 36 (2) (2002) 157-168.

[27] S.W. Doebling, C.R. Farrar, M.B. Prime, A summary review of vibration-based damage identification methods, The Shock and Vibration Digest 30 (2) (1998) 91-105.

[28] A. Joshi, B.S. Madhusudhan, A unified approach to free vibration of damaged beams having various homogeneous boundary conditions, Journal of Sound and Vibration 147 (3) (1991) 475-488.

[29] V. Pagneux, Multimodal admittance method in waveguides and singularity behavior at high frequencies, Journal of Computational and Applied Mathematics 234 (2010) 1834-1841.

[30] J. Schiff, S. Shnider, A natural approach to the numerical integration of Ricatti differential equations, SIAM (Soc. Ind. Appl. Math.) Journal on $550 \quad$ Numerical Analysis 36 (5) (1999) 1392-1413.

[31] Y. Y. Lu, A fourth-order magnus scheme for helmholtz equation, Journal of Computational and Applied Mathematics 173 (2005) 247-258. 
[32] A. Iserles, A. Martinsen, S.P. Norset, On the implementation of the method of magnus series for linear differential equations, BIT 39 (2) (1999) 281-304.

[33] Z. Wang, A.N. Norris, Waves in cylindrical shells with circumferential submembers : a matrix approach, Journal of Sound and Vibration 181 (3) (1995) 457-484.

[34] V.V. Krylov, F.J.B.S. Tilman, Acoustic 'black holes' for flexural waves as effective vibration dampers, Journal of Sound and Vibration 274 (3-5) (2004) 605-619.

[35] P.A. Feurtado, S.C. Conlon, F. Semperlotti, A normalized wave number variation parameter for acoustic black hole design, The Journal of the Acoustical Society of America 136 (2) (2014) EL148-152.

565 [36] V.V. Krylov, Propagation of localized vibration modes along edges of immersed wedge-like structures : geometrical-acoustics approach, Journal of Computational Acoustics 7 (1) (1999) 57-70. 